\title{
Clinical Study of Gefitinib for Non-Small Cell Lung Cancer
}

\author{
Yang $\mathbf{L i}^{*}$ \\ Taizhou Second People's Hospital, Taizhou 225500, Jiangsu Province, China \\ *Corresponding author: Yang Li, li19780824@163.com
}

\begin{abstract}
Objective: To evaluate the clinical efficacy of gefitinib in non-small cell lung cancer. Methods: A total of 45 patients with non-small cell lung cancer who received treatment in Taizhou Second People's Hospital from January 2018 to January 2020 were selected as the subjects in this study, in which all of them were treated with gefitinib. Its efficacy and safety were evaluated. Results: The objective response rate was $53.33 \%$ and the disease control rate was $84.44 \%$. After treatment, the levels of tumor markers were measured again, of which the levels of CA125 and CEA were significantly lower than before $(P<0.05)$. After treatment, the patients' $\mathrm{CD}_{3}{ }^{+}, \mathrm{CD}_{4}{ }^{+}$, and $\mathrm{CD}_{4}{ }^{+} / \mathrm{CD}_{8}{ }^{+}$were significantly lower than those before treatment, and $\mathrm{CD}_{8}{ }^{+}$was significantly higher $(P<0.05)$. Conclusion: Gefitinib, which is a targeted therapy for non-small cell lung cancer, can reduce the level of serum tumor markers and improve the immune function. The curative effect is good, but more emphasis should be on the adverse reactions caused by a single drug use.
\end{abstract}

Keywords: Gefitinib; Targeted therapy; Non-small cell lung cancer

Publication date: November 2021; Online publication: November 30, 2021

\section{Introduction}

Lung cancer is a common malignant tumor in China and even around the world. It is of high prevalence and high mortality. It is a serious threat to the life of people. Among various types of lung cancer, nonsmall cell lung cancer accounts for about $80 \%$ of the clinical incidence, and the vast majority of patients have already progressed to middle or advanced stage during clinical diagnosis ${ }^{[1]}$, thus missing the right time for radical treatment. The long-term survival rate of patients with advanced non-small cell lung cancer is very low. At this stage, surgical treatment is ineffective. Radiotherapy and chemotherapy can only inhibit metastasis and control the progress of the disease as much as possible in order to prolong the survival time. However, at present, traditional radiotherapy and chemotherapy methods can no longer meet the needs of patients, and their quality of life is affected by many adverse reactions, such as nausea, vomiting, hair loss, and even complications, such as liver and kidney failure ${ }^{[2]}$. Therefore, new, safe, and effective drugs need to be developed and explored. Based on the clinical development and wide application of molecular biology in recent years, targeted drugs have also achieved remarkable results in the treatment of non-small cell lung cancer. Gefitinib, as a new oral epidermal growth factor receptor tyrosine kinase inhibitor, has a positive effect on the inhibition of angiogenesis ${ }^{[3]}$. Taizhou Second People's Hospital has started to use gefitinib in the treatment of non-small cell lung cancer with remarkable results.

\section{Data and methods}

\subsection{Basic data}

A total of 45 patients with non-small cell lung cancer who were treated in Taizhou Second People's Hospital 
from January 2018 to January 2020 were selected as the subjects in this study. The inclusion criteria were as follows: (1) definite pathological or cytological diagnosis; (2) advanced pathological stage IV; (3) no abnormalities in blood routine examination, liver and kidney function, as well as electrocardiogram before admission; (4) no brain metastasis; (5) estimated survival time $>3$ months. The exclusion criteria were as follows: (1) tumors involving other systems, immune system diseases, brain and bone metastasis; (2) allergic to the drug used in this study protocol; (3) occurrence of serious complications, interruption, or having to switch to other drugs; (4) severe anemia and important organ dysfunction; (5) history of immunosuppressant or glucocorticoid use in the recent three months.

There were 24 male patients and 21 female patients. The age of the patients ranged from 52 years old to 77 years old, with an average age of $65.34 \pm 7.28$. Staging of the disease: 30 stage III patients and 15 stage IV patients. Pathological types: 37 cases of adenocarcinoma, 7 cases of squamous carcinoma, and a case of adenosquamous carcinoma.

\subsection{Therapeutic schedule}

Gefitinib was taken orally $250 \mathrm{mg} / \mathrm{time}$, qd, on an empty stomach or with food, and continued to be administered for 4 cycles, with 28 days as a treatment cycle. The following events were taken as the criteria for drug withdrawal: (1) the occurrence of intolerable toxic and side effects; (2) disease progression; (3) refusal of treatment; (4) death. In case of serious rash or diarrhea during treatment, the medication was suspended or taken orally every other day while providing symptomatic treatment.

\subsection{Observation indicators}

\subsubsection{Overall efficacy}

The overall efficacy of the treatment was evaluated based on the evaluation criteria of solid tumors: (1) complete remission (disappearance of target lesion); (2) partial remission (the length and diameter of the baseline lesion reduced by more than 30\%); (3) stable disease (the length and diameter of the baseline lesion decreased but did not meet the criteria for partial remission); (4) disease progression (the length and diameter of the baseline lesion increased by more than $20 \%$, or the appearance of new lesions). The objective response rate is taken as the sum of complete remission rate and partial remission rate. The disease control rate is taken as the sum of complete remission rate, partial remission rate, and stable disease rate ${ }^{[4]}$.

\subsubsection{Tumor markers}

Before and after treatment, fasting venous blood was drawn from each patient in the morning, and the serum was stored for testing after centrifugation. It was placed at a temperature of $-80^{\circ} \mathrm{C}$. The level of carbohydrate antigen 125 (CA125) was detected by enzyme-linked immunosorbent assay (ELISA) for 3 consecutive times and the average value was taken. The level of carcinoembryonic antigen (CEA) was detected by magnetic particle-based chemiluminescence enzyme immunoassay for 3 consecutive times and the average value was calculated.

\subsubsection{T-lymphocyte count}

The T-lymphocyte count was measured by flow cytometry before and after treatment: $\mathrm{CD}_{3}{ }^{+}, \mathrm{CD}_{4}{ }^{+}, \mathrm{CD}_{8}{ }^{+}$, and $\mathrm{CD}_{4}{ }^{+} / \mathrm{CD}_{8}{ }^{+}$.

\subsubsection{Adverse reactions}

The adverse reactions related to the treatment in the course of gefitinib were monitored, such as diarrhea, 
anemia, rash, etc.

\subsection{Statistical analysis}

The statistical data processing software, SPSS 25.0, was used. The counting data were expressed as percentage data and were tested by $\mathrm{X}^{2}$ test. In addition, the measurement data expressed in the form of mean \pm standard deviation were tested by t-test. $P<0.05$ indicates that the results of the intergroup test is statistically significant.

\section{Results}

\subsection{Efficacy evaluation}

Among the 45 patients, 2 patients (4.44\%) had complete remission, 22 patients $(48.89 \%)$ had partial remission, 14 patients $(31.11 \%)$ were of stable disease, and there were 7 cases $(15.56 \%)$ with disease progression. The objective response rate was $53.33 \%$ (24/45), and the disease control rate was $84.44 \%$ $(38 / 45)$.

\subsection{Tumor markers}

After treatment, the levels of tumor markers were measured again, of which the levels of CA125 and CEA were significantly lower than before treatment $(P<0.05)$ (Table 1).

Table 1. The levels of tumor markers before and after treatment $(\bar{x} \pm s)$

\begin{tabular}{ccc}
\hline Time & CA125 $(\mathrm{U} / \mathrm{ml})$ & CEA $(\mathrm{ng} / \mathrm{ml})$ \\
\hline Before treatment & $64.01 \pm 8.72$ & $26.12 \pm 1.98$ \\
After treatment & $20.26 \pm 2.37$ & $15.09 \pm 1.41$ \\
$\mathrm{t}$ & 32.478 & 30.440 \\
$P$ & 0.000 & 0.000 \\
\hline
\end{tabular}

\subsection{T-lymphocyte count}

After treatment, the $\mathrm{CD}_{3}{ }^{+}, \mathrm{CD}_{4}{ }^{+}$, and $\mathrm{CD}_{4}{ }^{+} / \mathrm{CD}_{8}{ }^{+}$counts were significantly lower than those before treatment, but the $\mathrm{CD}_{8}{ }^{+}$count was significantly higher after treatment $(P<0.05)$ (Table 2).

Table 2. T-lymphocyte count before and after treatment $(\bar{x} \pm \mathrm{s}, \%)$

\begin{tabular}{ccccc}
\hline Time & $\mathrm{CD}_{3}{ }^{+}$ & $\mathrm{CD}_{4}{ }^{+}$ & $\mathrm{CD}_{8}{ }^{+}$ & $\mathrm{CD}_{4}{ }^{+} / \mathrm{CD}_{8}{ }^{+}$ \\
\hline Before treatment & $59.03 \pm 5.87$ & $38.11 \pm 2.73$ & $30.87 \pm 3.45$ & $1.23 \pm 0.49$ \\
After treatment & $42.65 \pm 4.18$ & $29.63 \pm 2.14$ & $33.52 \pm 4.13$ & $0.80 \pm 0.15$ \\
$t$ & 15.248 & 16.399 & 3.303 & 5.629 \\
$P$ & 0.000 & 0.000 & 0.000 & 0.000 \\
\hline
\end{tabular}

\subsection{Adverse reactions}

The adverse reactions were observed during the course of treatment: 5 cases of diarrhea, 2 cases of anemia, a case of rash, a case of abnormal coagulation, and a case of abnormal liver function; there were 10 cases in total which developed adverse reactions. The incidence of adverse reactions was $22.22 \%$. 


\section{Discussion}

Gefitinib is a targeted therapeutic drug commonly used in clinic. It blocks phosphorylation by inhibiting the pathway of tyrosine kinase associated with the epidermal growth factor receptor, inhibits the proliferation and metastasis of cancer cells, induces cancer cell apoptosis, and prevents angiogenesis in cancer, thus playing an anti-cancer effect ${ }^{[5]}$.

Serum tumor markers can reflect the tumor status in patients. Among various markers, CA125 is a mixed marker, which is mainly expressed in epithelial cells and tumors. Real-time monitoring of the changes in the level of this index can effectively reflect the therapeutic effect. CEA is an acidic glycoprotein with embryonic antigen specificity. The changes in this index's level can reflect the progress and metastasis of the disease to a certain extent. The concentration of CEA gradually increases in the process of cell transformation from symptom to malignancy ${ }^{[6]}$. The results of this study showed that the levels of CEA and CA125 of the patients after treatment were significantly lower than those before treatment, suggesting that gefitinib has a good effect.

As patients with non-small cell lung cancer have impaired immune function, the protection of their immune function is also a key to clinical treatment. T-lymphocytes regulate the cellular immune process and participate in anti-cancer immunity. With a decline in the function of T-lymphocytes, the occurrence and progress of cancer cells would be promoted. Among them, $\mathrm{CD}_{3}{ }^{+}$and $\mathrm{CD}_{4}{ }^{+}$can enhance cellular immunity, while $\mathrm{CD}_{8}{ }^{+}$can inhibit cellular immune process ${ }^{[7]}$. This study has observed that after treatment, $\mathrm{CD}_{3}{ }^{+}, \mathrm{CD}_{4}{ }^{+}$, and $\mathrm{CD}_{4}{ }^{+} / \mathrm{CD}_{8}{ }^{+}$were significantly lower than those before treatment, but the $\mathrm{CD}_{8}{ }^{+}$count was significantly higher, suggesting that gefitinib can regulate the expression of T-lymphocytes in patients with non-small cell lung cancer and improve their immune function.

The adverse reactions of patients who have been treated with gefitinib include diarrhea, anemia, and rare liver function damage as observed in this study. There is still a need to continue to pay attention to the adverse reactions caused by gefitinib ${ }^{[8]}$.

In conclusion, gefitinib, which is used as a targeted therapy for non-small cell lung cancer, can reduce the level of serum tumor markers and improve immune function. Its curative effect is good, and it is necessary to pay attention to the adverse reactions caused by a single drug use.

\section{Disclosure statement}

The author declares that there is no conflict of interest.

\section{References}

[1] Zhang Y, Zhang HR, Tie XJ, et al., 2021, Effects of Gefitinib on MMP-9, TIMP-1 and Prognosis in Elderly Patients with advanced EGFR Mutant Non-small Cell Lung Cancer. Chinese Journal of Gerontology, 9(9) : 1821-1824.

[2] Zhang BS, Zhang Y, Zhao CY, et al., 2021, Effect of Gefitinib Combined with Three-dimensional Conformal Radiotherapy on Elderly Patients with Advanced Non-small Cell Lung Cancer and Its Effect on Serum Tumor Markers. Medical \& Pharmaceutical Journal of Chinese People's Liberation Army, 33(2): 14-17.

[3] Cai HF, Xu HB, 2021, Effects of Second-line Gefitinib on the Efficacy and Survival of Patients with Advanced Non-small Cell Lung Cancer at Different Epidermal Growth Factor Receptor Mutation Sites. Oncology Progress, 19(13): 1338-1340, 1344.

[4] Ma LH, Han YJ, 2021, Clinical Efficacy and Safety of Gefitinib in Patients with Advanced Non-small Cell Lung Cancer. Global Traditional Chinese Medicine, 14(4): 11-12, 17. 
[5] Jin CY, 2021, Therapeutic Effect of Gefitinib and Chemotherapy on Patients with Advanced NonSmall Cell Lung Cancer. Contemporary Medicine, 27(17): 38-40.

[6] Peng J, 2021, Pharmaconursing Association of 256 Patients with Non-small Cell Lung Cancer Treated with Gefitinib. Modern Chinese Doctor, 59(5): 8-11.

[7] Sun YH, He QY, Guo QY, et al., 2021, Research of Gefitinib Targeted Therapy on Quality of Life in Patients with Advanced Non-small Cell Lung Cancer. Health Required, (11): 235.

[8] Shen L, Zhu YY, Liu ZG, et al., 2021, Efficacy of Gefitinib in Targeted Therapy of Advanced Nonsmall Cell Lung Cancer. Health Required, (22): 104-105. 